04

\title{
Формирование микроканальной структуры искрового разряда в воздухе в промежутке „острие-плоскость“"
}

\author{
() А.А. Тренькин, К.И. Алмазова, А.Н. Белоногов, В.В. Боровков, Е.В. Горелов, И.В. Морозов, С.Ю. Харитонов \\ Российский Федеральный ядерный центр Всероссийский научно-исследовательский институт экспериментальной \\ фозики, \\ 607190 Саров, Россия \\ e-mail: alexey.trenkin@gmail.com
}

Поступило в Редакцию 12 августа 2021 г.

В окончательной редакции 25 ноября 2021 г.

Принято к публикации 26 ноября 2021 г.

\begin{abstract}
Методом теневого фотографирования исследовано формирование микроканальной структуры искрового разряда в промежутке „острие (катод)-плоскость“ длиной $1.5 \mathrm{~mm}$ в воздухе атмосферного давления. Зарегистрированы изображения с последовательным уменьшением диаметров микроканалов и увеличением их количества в прикатодной области на временном интервале $5 \mathrm{~ns}$. Полученные данные интерпретированы в рамках механизма формирования микроструктуры за счет неустойчивости фронта волны ионизации. Выполнены оценки параметров, характеризующих процесс образования микроструктуры, и получено их удовлетворительное согласие с экспериментальными данными.
\end{abstract}

Ключевые слова: искровой разряд, ионизационная неустойчивость, микроструктура, метод теневого фотографирования.

DOI: $10.21883 /$ JTF.2022.03.52130.235-21

\section{Введение}

Востребованность практических применений электрических разрядов в плотных газах стимулирует интерес к их изучению для повышения эффективности существующих и развития новых газоразрядных технологий. Несмотря на длительный период исследований и большой объем полученных данных, касающихся различных аспектов газоразрядных процессов, ряд явлений до сих пор слабо изучен. Одним из них является формирование микроструктуры в начальной фазе разрядов в воздухе атмосферного давления, когда канал представляет собой совокупность большого числа микроканалов (филаментов) [1-7]. Изначально микроструктура разряда была обнаружена методом автографов (отпечатков) на поверхности плоского электрода (см. [5-7] и ссылки в них). Микроканальная структура в объеме разрядного промежутка была зарегистрирована относительно недавно с применением метода лазерного зондирования и основанных на нем теневых и интерференционных методиках [1-4]. Сложность экспериментального исследования данного явления определяется необходимостью обеспечения высокой разрешающей пространственной (микрометрового уровня) и временной (нано- и субнаносекундного уровня) способности диагностики. Отметим, что оптическими и электроннооптическими методами микроструктура была не разрешима [1].

Вместе с тем, если факт наличия микроструктуры исследованных разрядов не вызывает сомнения, то вопрос о механизме ее формирования до сих пор остается открытым.
Одной из возможных причин филаментации разряда может являться ионизационно-перегревная неустойчивость, развитием которой объясняется, например, формирование множества нитевидных каналов диаметром от 60 до $100 \mu \mathrm{m}$ в изначально однородном диффузном разряде в аргоне при давлении 300 Torr в промежутке острие-плоскость длиной $3 \mathrm{~mm}$ [8]. Однако там формирование микроструктуры регистрировалось спустя $100-400 \mathrm{~ns}$ от начала пробоя, в отличие от условий экспериментов [1-4], где микроканалы регистрируются уже на первых наносекундах после пробоя. Оценки показывают, что за столь короткое время развитие ионизационно-перегревной неустойчивости в условиях рассматриваемых экспериментов маловероятно [6].

Более подходящим является объяснение формирования филаментов за счет неустойчивости фронта волны ионизации, о чем свидетельствует ряд расчетнотеоретических работ [6,7,9-13]. Вместе с тем до настоящего времени этому отсутствует достоверное экспериментальное подтверждение, что может быть связано со сложностью регистрации процессов в фазе распространения волны ионизации. Данное обстоятельство обусловлено как короткой длительностью этой фазы, так и относительно низкими значениями концентрации электронов и градиентом плотности газа в этот момент, что не позволяет регистрировать микроструктуру используемыми методиками [1-4].

В работах $[1,2]$ была реализована методика исследования пространственной структуры разряда методом теневого фотографирования с пространственным разрешением $5 \mu \mathrm{m} / 3$ pixel при длительности лазерного импульса на полувысоте 6 ns. В этих исследованиях была 


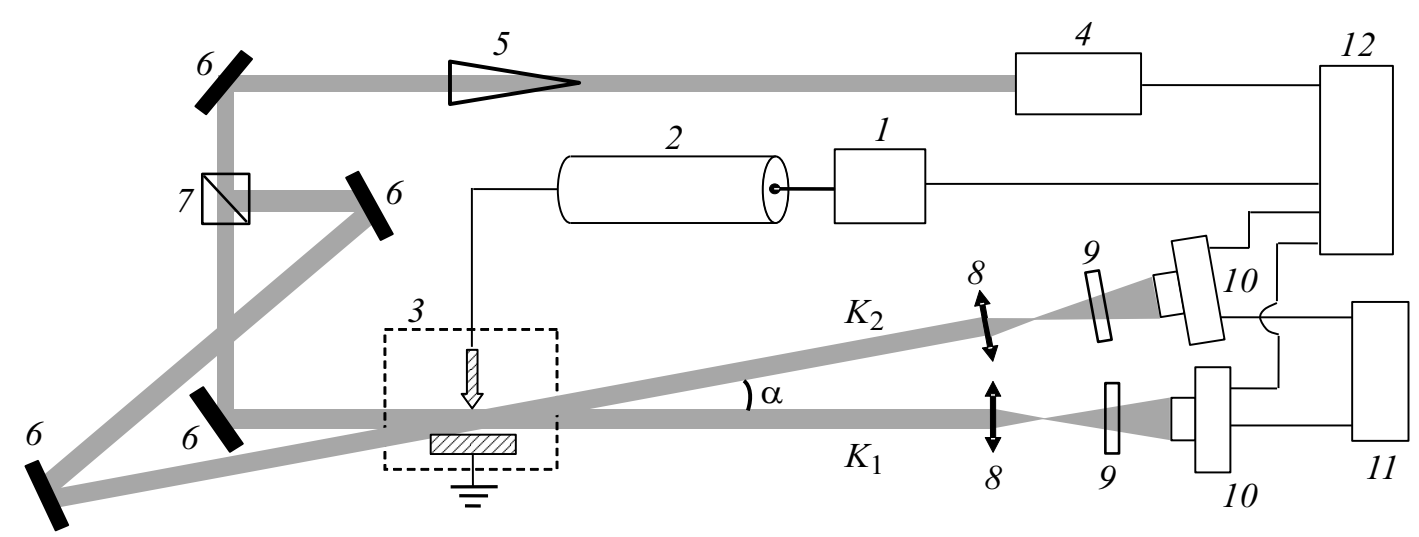

Рис. 1. Принципиальная схема экспериментального стенда: 1 - генератор импульсов напряжения, $2-$ кабельная линия, 3 - разрядный промежуток, 4 - источник зондирующего сигнала (лазер), 5 - коллиматор, 6 - поворотное зеркало, 7 - светоделительный элемент, 8 - объектив, 9 - светофильтры, 10 - электронно-оптический регистратор, 11 - персональный компьютер, 12 - блок синхронизации. Угол $\alpha-$ между лазерными каналами $K_{1}$ и $K_{2}$ составляет $\alpha=15^{\circ}$.

зарегистрирована микроканальная структура искрового разряда в воздухе атмосферного давления в промежутке „острие-плоскость“ на начальной стадии его развития. Установлено, что канал представляет собой пучок большого числа микроканалов. На временах от единиц до десятков ns происходит развитие микроканалов от острия вглубь разрядного промежутка и их расширение. Затем наблюдается формирование общего фронта цилиндрической ударной волны канала искры и его радиальное движение. Существенным недостатком данной методики являлась однокадровость, что ограничивало возможности исследования динамики разряда.

Настоящая работа является продолжением исследований $[1,2]$. Здесь в развитие методики теневого фотографирования реализован ее двухкадровый вариант, что значительно расширяет возможности получения данных о процессах, сопровождающих газовые разряды. В работе представлены результаты исследования формирования микроструктуры в искровом разряде.

\section{1. Экспериментальная аппаратура и методика}

Схема экспериментального стенда представлена на рис. 1. Часть элементов схемы подробно описаны в $[1,2]$. Генератор импульсов напряжения обеспечивал на выходе импульс отрицательной полярности амплитудой $25 \mathrm{kV}$ и длительностью фронта по уровню $0.1-0.9$ около $7 \mathrm{~ns}$. Через кабельную линию импульс подавался на разрядный промежуток. Измерения напряжения и тока осуществлялись на выходе генератора импульсов напряжения соответственно емкостным делителем и резистивным шунтом. Временное разрешение делителя и шунта - менее $1 \mathrm{~ns}$. Регистрация сигналов производилась осциллографом с полосой пропускания $500 \mathrm{MHz}$ и скоростью оцифровки $2 \mathrm{Gs} / \mathrm{s}$.

Электродная система имела геометрию „острие-плоскость“. Осесимметричный острийный электрод изготов- лен из нержавеющей стали и имел длину $19 \mathrm{~mm}$, диаметр $14 \mathrm{~mm}$, угол при вершине $36^{\circ}$ и радиус кривизны $0.15 \mathrm{~mm}$. В качестве плоского электрода использовался электрод, изготовленный из алюминиевого сплава с рабочей частью, близкой по форме к шаровому сегменту диаметром $4.5 \mathrm{~cm}$ толщиной $1.5 \mathrm{~cm}$. Межэлектродный зазор составлял $1.5 \mathrm{~mm}$.

В составе стенда использовалась система оптической регистрации разряда. Система включала в себя источник зондирующего излучения - твердотельный лазер (длина волны $532 \mathrm{~nm}$, длительность импульса на полувысоте $6 \mathrm{~ns}$ ), объектив, светофильтры и цифровую электронно-оптическую камеру. Плоскопараллельный пучок лазерного излучения, проходя через область разряда перпендикулярно оси электрода-острия, регистрировался электронно-оптической камерой. В области формирования разряда поперечный размер лазерного пучка составлял примерно $1 \mathrm{~cm}$ и имел гауссов профиль. Поскольку межэлектродный зазор значительно меньше размера пучка, это обеспечивало достаточно однородное поле лазерного излучения в области разряда.

На базе этой системы реализована методика теневого фотографирования. В настоящих экспериментах в отличие от проведенных ранее $[1,2]$ для теневой методики использовалась двухлучевая оптическая схема, что обеспечивало получение двух кадров за импульс. Угол между лазерными лучами составлял около $15^{\circ}$. Для каждого луча изображение в области разрядного промежутка строилось с помощью объектива с фокусным расстоянием $23 \mathrm{~cm}$ на фотокатоде электронно-оптического регистратора. Коэффициент увеличения равен десяти. Экспозиция каждого кадра определялась длительностью импульса лазера. Изменением разности оптической длины пути лучей можно было варьировать временной интервал между кадрами тенеграмм. В экспериментах этот интервал составлял $5 \mathrm{~ns}$. Временная привязка кадров осуществлялась относительно момента пробоя, а характеризующее их время соответствует началу кадра. 

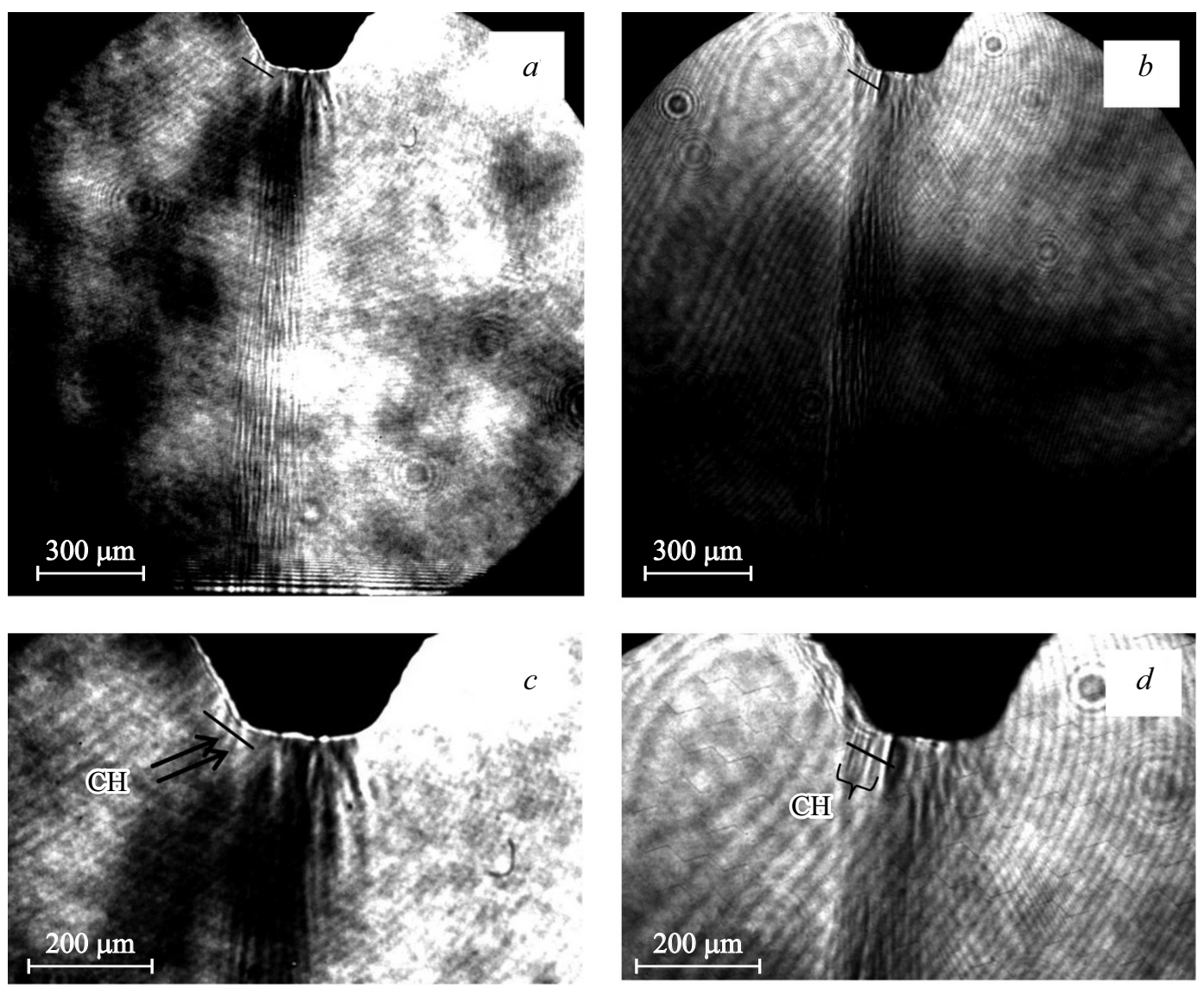

Рис. 2. Тенеграммы разряда в одном импульсе в момент времени $9(a)$ и $14 \mathrm{~ns}(b) ; c$ и $d-$ соответствующие увеличенные фрагменты. Черная линия ( $a, c$ - длина $120 \mu \mathrm{m}, b, d-90 \mu \mathrm{m})$, пересекающая каналы СН, обозначает путь, вдоль которого строилась степень почернения изображения.

Сдвигом момента запуска лазера и электроннооптического регистратора относительно момента пробоя обеспечивалась визуализация различных стадий разрядного процесса. Разрешающая способность оптической системы составляла $5 \mu \mathrm{m} / 3$ pixel.

\section{2. Экспериментальные результаты и их обсуждение}

Разряд исследовался в воздухе при нормальных условиях. После пробоя промежутка в разрядной цепи возникал колебательный процесс с экспоненциальным затуханием тока и напряжения. Период колебаний составлял $0.6 \mu \mathrm{s}$, амплитуда тока и время его затухания соответственно $1 \mathrm{kA}$ и $1.2 \mu \mathrm{s}$. При этом на осциллограммах выделялись два характерных момента времени: появление напряжения на разрядном промежутке и пробой. Среднее значение задержки между ними - $4 \mathrm{~ns}$. За момент пробоя $t_{b d}$ был принят момент начала роста тока и соответственно спада напряжения.

Как свидетельствуют результаты проведенных ранее исследований $[1,2]$, динамика разряда в начальной фазе представляется следующей. В предпробойной стадии возникает слабосветящийся диффузный канал и катод- ные пятна, которые дают начало искровому каналу. Канал обладает микроструктурой, которая регистрируется на тенеграммах уже в первые наносекунды после пробоя. До момента 15-20 ns диаметр искры практически не меняется, ток нарастает, а концентрация электронов в этот момент достигает максимального значения, после чего происходит радиальное расширение канала с образованием на его границе цилиндрической ударной волны.

Во введении была отмечена проблематичность регистрации процесса формирования микроструктуры: высокие скорости, обусловленные значительным перенапряжением разрядного промежутка в предпробойной фазе, и относительно низкие для регистрации значения концентрации электронов и градиентов плотности газа. Однако после формирования канала разряда зачастую наблюдается образование запаздывающих боковых каналов, берущих свое начало на смещенных от осевой области зонах катода. В этой фазе процессы развиваются при меньших электрических полях, что обусловливает их более низкую скорость.

Полученные тенеграммы (рис. 2) обнаружили вблизи боковой поверхности острийного электрода каналы, развивающиеся после формирования основного канала, а использование в настоящих экспериментах двухкад- 
рового режима теневого фотографирования позволили зарегистрировать их динамику.

На тенеграмме (рис. 2, a, c) присутствуют два канала, проявляющие себя в виде темных вытянутых областей. Черная линия ( $a, c$ - длина $120 \mu \mathrm{m}, b, d-90 \mu \mathrm{m})$, пересекающая каналы $\mathrm{CH}$, обозначает путь, вдоль которого строился профиль степени почернения изображения. На рис. 3 представлен данный профиль. Цифрами 1 и 2 обозначены характерные минимумы. Также на рис. 3 приведен профиль степени почернения в отсутствии разряда. Сравнение двух профилей показывает, что темные области на тенеграмме, возникают именно в разряде и не связаны с особенностями поля визуализации, что подтверждает их идентификацию в качестве каналов. Их диаметры, определенные как ширина на полувысоте профиля, составляют $10-15 \mu \mathrm{m}$.

Тенеграмма (рис. $2, b, d$ ), полученная с интервалом $5 \mathrm{~ns}$ после тенеграммы на рис. 2, a, , обнаруживает на месте нахождения первоначальных каналов несколько каналов меньшего диаметра. Соответствующий профиль степени почернения приведен на рис. 4. Минимумы, предположительно соответствующие каналам, обозначены на этом рисунке цифрами от 1 до 4. Исходя из этого, можно полагать, что количество каналов равно четырем. Обращает на себя внимание общее снижение яркости изображения в этой области по сравнению с картиной без разряда. Кроме того, заметна модуляция профиля. По-видимому, это связано с частичным перекрытием каналов на линии луча лазера. Определенные, как и выше, диаметры каналов составляют от 5 до $10 \mu \mathrm{m}$, что примерно в два раза меньше, чем диаметры исходных каналов.

Обнаруженные особенности динамики газоразрядной структуры, включающие формирование каналов с последовательным уменьшением диаметра и ростом их количества, позволяют рассматривать данное явление как результат развития неустойчивости ионизационного фронта. Соответствующая модель механизма формирования микроструктуры разряда рассматривалась ранее в [6,7]. На основании этой модели выполним оценки для условий настоящих экспериментов, полагая, что каналы, регистрируемые на тенеграмме рис. $2, b, d$, сформировались в результате ионизационной неустойчивости фронта каналов, представленных на тенеграмме рис. $2, a, c$.

Используя данные по чувствительности применяемой методики, можно полагать, что концентрация электронов в каналах составляет не менее $10^{16} \mathrm{~cm}^{-3}$ [14]. Столь высокое значение концентрации свидетельствует о том, что развитие неустойчивости происходит не в лавинной, а в плазменной фазе [7]. Для рассматриваемых условий удельное сопротивление плазмы канала составляет $\rho \leq 1 \Omega \mathrm{m}$, тогда максвелловское время релаксации заряда $\tau_{M}=\varepsilon_{0} \rho \leq 10^{-11} \mathrm{~s}$ не превосходит характерного времени ионизации воздуха электронным ударом $\tau_{e} \approx 10^{-11} \mathrm{~s}$ в рассматриваемых полях [15]. Данное обстоятельство позволяет использовать приближение идеальной проводимости плазмы для интересующих нас

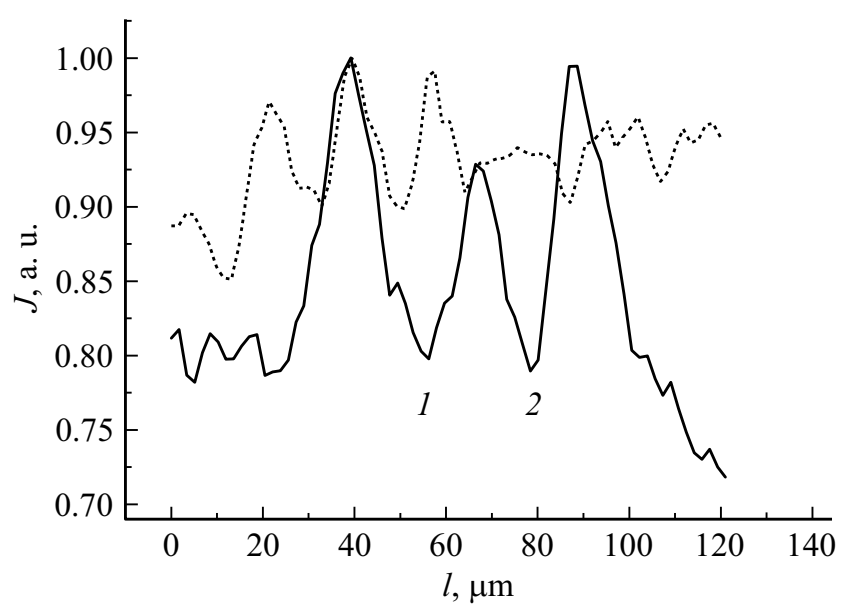

Рис. 3. Профиль степени почернения $J$ изображения тенеграммы вдоль выделенного на рис. 2, a, $c$ пути $l$. Штриховая линия - в отсутствие разряда, сплошная линия - при разряде.

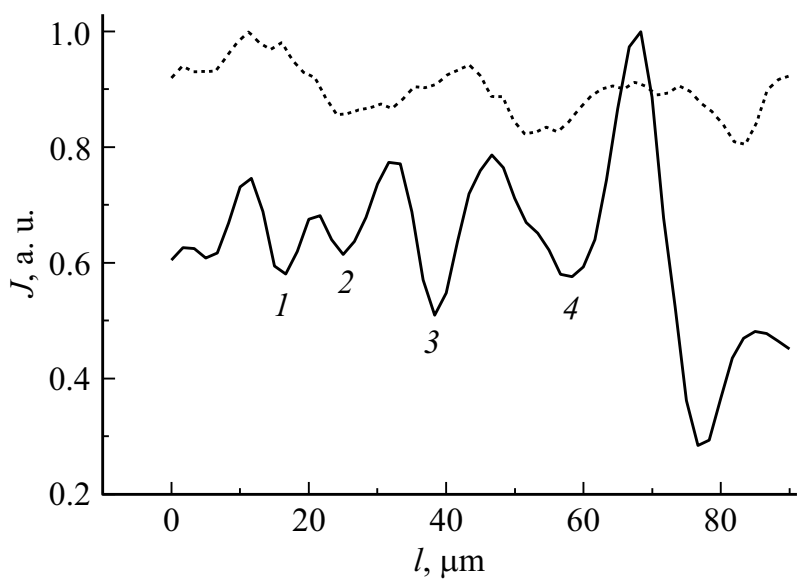

Рис. 4. Профиль степени почернения $J$ изображения тенеграммы вдоль выделенного на рис. $2, b, d$ пути $l$. Штриховая линия - в отсутствие разряда, сплошная линия - при разряде.

процессов, и оценка времени развития неустойчивости на головке канала (фронте ионизации) дает [9]:

$$
t_{\text {ins }}=\lambda / \mu E,
$$

где $\lambda$ - длина волны возмущения потенциала на фронте ионизации, $\mu$ - подвижность электронов, $E-$ напряженность электрического поля. Полагая для наших условий $E=100-300 \mathrm{kV} / \mathrm{cm}, \lambda=10-20 \mu \mathrm{m}$, имеем $t_{\mathrm{ins}} \approx 10 \mathrm{ps}$. Поскольку $t_{\mathrm{ins}}$ порядка $\tau_{e}$ можно полагать, что размер формирующихся микроканалов $l_{m}$ должен определяться величиной порядка $\alpha^{-1}$, где $\alpha-$ коэффициент ионизации Таунсенда. Используя для $\alpha$ формулу [16]:

$$
\alpha=A p \exp (-B p / E),
$$

где $p$ - давление газа, $A$ и $B-$ константы, для рассматриваемых условий, получаем значение $l_{m}=2-15 \mu \mathrm{m}$, что соответствует зарегистрированным величинам. 
Таким образом, полученные в настоящей работе результаты свидетельствуют в пользу механизма формирования микроканальной структуры разряда за счет развития неустойчивости фронта ионизации.

\section{Заключение}

С использованием двухкадровой методики теневого фотографирования исследован искровой разряд в промежутке „острие (катод)-плоскость“ длиной $1.5 \mathrm{~mm}$ в воздухе атмосферного давления.

Проведенные исследования подтвердили полученные ранее с помощью однокадровой методики данные о динамике разряда на временах от момента пробоя до $100 \mathrm{~ns}$, включающей: развитие микроканалов от острия вглубь разрядного промежутка, расширение микроканалов, формирование общего фронта канала искры, цилиндрической ударной волны и его радиальное движение.

Зарегистрирована динамика развития боковых каналов в прикатодной области во временном диапазоне от 9 до $14 \mathrm{~ns}$, включающая последовательное формирование каналов меньшего диаметра и рост их количества. Диаметр исходного канала составляет 10-15 $\mu \mathrm{m}$, диаметр последующих каналов - 5-10 $\mu \mathrm{m}$, при этом наблюдается удвоение количества каналов на масштабе времени, не превышающем $5 \mathrm{~ns}$.

Полученные экспериментальные данные подтверждают предположение о механизме формирования микроканальной структуры за счет неустойчивости ионизационного фронта. Выполнены оценки параметров, характеризующих процесс формирования микроструктуры, которые согласуются с экспериментальными данными и высказанным предположением.

\section{Конфликт интересов}

Авторы заявляют, что у них нет конфликта интересов.

\section{Список литературы}

[1] А.А. Тренькин, К.И. Алмазова, А.Н. Белоногов, В.В. Боровков, Е.В. Горелов, И.В. Морозов, С.Ю. Харитонов. ЖТФ, 8 (6), 827 (2018).

DOI: 10.21883/JTF.2018.06.46011.2511 [A.A. Trenkin, K.I. Almazova, A.N. Belonogov, V.V. Borovkov, E.V. Gorelov, I.V. Morozov, S.Yu. Kharitonov. Tech. Phys., 63 (6), 801 (2018). DOI: 10.1134/S1063784218060026]

[2] А.А. Тренькин, К.И. Алмазова, А.Н. Белоногов, В.В. Боровков, Е.В. Горелов, И.В. Морозов, С.Ю. Харитонов. ЖТФ, 90 (12), 2039 (2020).

DOI: $10.21883 /$ JTF.2020.12.50119.435-19 [A.A. Trenkin, K.I. Almazova, A.N. Belonogov, V.V. Borovkov, E.V. Gorelov, I.V. Morozov, S.Yu. Kharitonov. Tech. Phys., 65 (12), 1948 (2020). DOI: 10.1134/S1063784220120270]

[3] E.V. Parkevich, M.A. Medvedev, A.I. Khirianova, G.V. Ivanenkov, A.S. Selyukov, A.V. Agafonov, K.V. Shpakov, A.V. Oginov. Plasma Sources Sci. Technol., 28, 125007 (2019). DOI: 10.1088/1361-6595/ab518e
[4] E.V. Parkevich, M.A. Medvedev, G.V. Ivanenkov, A.I. Khirianova, A.S. Selyukov, A.V. Agafonov, Ph.A. Korneev, S.Y. Gus'kov, A.R. Mingaleev. Plasma Sources Sci. Technol., 28, 095003 (2019). DOI: 10.1088/1361-6595/ab3768

[5] А.Г. Репьев, П.Б. Репин, В.С. Покровский. ЖТФ, 77 (1), 56 (2007). [A.G. Rep'ev, P.B. Repin, V.S. Pokrovski'. Tech. Phys., 52 (1), 52 (2007).]

[6] В.И. Карелин, А.А. Тренькин. ЖТФ, 78 (3), 29 (2008). [A.A. Trenkin, V.I. Karelin. Tech. Phys., 53 (3), 314 (2008). DOI: 10.1134/S1063784208030055]

[7] В.И. Карелин, А.А. Тренькин. ЖТФ, 78 (9), 134 (2008). [V.I. Karelin, A.A. Trenkin. Tech. Phys., 53 (9), 1236 (2008). DOI: $10.1134 / \mathrm{S} 106378420809017 \mathrm{X}]$

[8] Ю.И. Бычков, Ф.И. Суслов, К.А. Тинчурин, А.Г. Ястремский. ФП, 17 (2), 196 (1991).

[9] Э.Д. Лозанский, О.Б. Фирсов. Теория искры (Атомиздат, М., 1975)

[10] О.А. Синкевич. ТВТ, 41 (5), 695 (2003).

[11] M. Arrayas, M. Fontelos, J. Trueba. Phys. Rev. Lett., 95 (5), 165001 (2005). DOI: 10.1103/PhysRevLett.95.165001

[12] A. Rocco, U. Ebert, W. Hundsdorfer. Phys. Rev. E, 66, 035102(R) (2002). DOI: 10.1103/PhysRevE.66.035102

[13] A. Luque, F. Brau, U. Eber. Phys. Rev. E, 78, 016206 (2008). DOI: 10.1103/PhysRevE.78.016206

[14] А.А. Тренькин, К.И. Алмазова, А.Н. Белоногов, В.В. Боровков, Е.В. Горелов, И.В. Морозов. ЖТФ, 91 (2), 255 (2021). DOI: 10.21883/JTF.2021.02.50359.217-20

[A.A. Trenkin, K.I. Almazova, A.N. Belonogov, V.V. Borovkov, E.V. Gorelov, I.V. Morozov, S.Yu. Kharitonov. Tech. Phys., 66 (2), 243 (2021). DOI: 10.1134/S1063784221020225]

[15] Э.М. Базелян, Ю.П. Райзер. Физика молнии и молниезащиты (Физматлит, М., 2001), ISBN 5-9221-0082-3

[16] Yu.P. Raiser. Physics of Gas Discharge (Intelligence, Dolgoprudny, 2009) 